\title{
Das geschlechtliche Verhältniss bei den nicht selbst brütenden Vögeln.
}

\author{
Von
}

\section{Dr. C. W. L. Gloger.}

Der Ausdruck, nicht selbst brütende ${ }^{*}$ ist, wie leicht zu erachten, zunächst im Sinne eines Gegensatzes zu jenen sehr wenigen Arten heisser Länder zu verstehen, deren Eier, seltsamer Weise, überhaupt gar nicht bebrütet werden und zu werden brauchen: da sie mit einer, nach Verhältniss ganz ausserordentlichen Grösse die wunderbare Eigenschaft verbinden, dass das Junge sich in ihnen, gleichwie in jenen der Amphibien, schon ohne Bebrüten entwickelt und, zum Leben gelangt, in gleicher Art selbst für sich zu sorgen versteht, ohne Schutz oder gar Pflege zu bedürfen. *)

So gering nun auch die Gesammtzahl solcher "nicht selbst brütender" Gattungen zu sein scheint, da sie ausser denen der Ku ckuksF amilie nur, so viel bisher bekannt, noch den $\mathrm{Kuhfinken} \mathrm{des} \mathrm{nörd-}$ lichen Amerika's umfasst: so wird jetzt bei dem erhöhten Interesse, welches die Fortpflanzungsgeschichte der ersteren durch neuere Beobachtungen gewonnen hat, nun doch bei beiden zugleich das.Verhältniss der Geschlechter zu einander gebührend in Betracht zu ziehen sein. Denn ohne Zweifel muss dasselbe jetzt schon an sich eine höhere Bedeutung gewinnen, um seine bereits erkennbare oder wahrscheinliche Beziehung auf das Uebrige dieser ungewöhnlichen Fortpflanzungsweise in beiden Fällen zu prüfen. Ganz besonders aber wird es nöthig bleiben, auch manche andere, beiderseits nebenher laufende Verhältnisse einer solchen vergleichenden Betrachtung zu unterziehen. Sonst könnte man, wie das leider überall so häufig geschieht, leicht wieder in Gefahr kommen, von der theilweisen Aehnlichkeit zu rasch auf durchgreifende Aehnlichkeit des Ganzen zu schliessen: auch da, wo eine solche, genauer erwogen, vielleicht alle Wahrscheinlichkeit gegen sich haben würde.

Betrachten wir, als höchst merkwürdig in dieser Hinsicht, zuvörderst jenen seltsamen Amerikaner,

den Kuhfinken, Fringilla pecoris Gm., Icterus pec. Bonap., Emberiza pec. Wils., Molothrus pec. Sws., Hypobletis pec. mihi.

Bei ihm findet, den übereinstimmenden Beobachtungen der nordamerikanischen Ornithologen zufolge, in Betreff der Verbindung beider Geschlechter mit einander stets ein so völlig ungeregeltes Verhältniss Statt, dass er hierin Seinesgleichen nicht hat. Denn wenn es darauf ankäme, nach einer treffenden und kurzen Bezeichnung für dasselbe zu suchen, (ähnlich Hrn. Brehm's höchst passendem Ausdrucke „zigeunerartiges Leben " für das, von keiner Jahreszeit abhängige, sondern bloss

*) Bekanntlich gehört aber dahin bloss die sehr geringzählige Familie der, halb hühner- und halb waderähnlichen Grossfusshüner der óstlichen Sunda-Inseln und Neuhollands, die Gattung Megapodius Temminck's. 
nach dem jeweiligen Vorhandensein oder Fehlen reichlicher Nahrung sich richtende Herumziehen, sich Ansiedeln, Fortpflanzen und Wiederfortwandern der Kreuzschnäbel:) dann würde hier vielleicht nur Ein solcher Ausdruck zu finden sein, welcher vollständig passte. Nämlich man würde dieses Verhältniss als krassesten „St. Simonismus, “ d. h. als ganz ungeordnete, daher stets beliebig wechselnde \#Gemeinschaft der Weiber," zu bezeichnen haben.

Wils on spricht auch hierüber theils nach eigenen Beobachtungen, theils nach Mittheilungen, die ihm von verschiedenen Seiten her zukamen; besonders nach den Berichten des Dr. Pott e r zu Baltimore. *)

"Dieser bemerkte, dass die Kuhvögel sich gar nicht paren: also dasselbe, was Dr. Jenner zu seiner Zeit hinsichtlich des Kuckuks beobachtet". **) Denn er sah diese Vögel während ihrer Fortpflanzungszeit in geraden und ungeraden $\mathrm{Zahlen,}$ von $1-20$, be i einander. Trennt sich ein Weibchen von der Gesellschaft: so wird seine Abwesenheit gar nicht bemerkt. Kein zärtlicher Gatte begleitet es, oder zeigt Besorgniss; auch wird seine Wiederkehr nicht mit jenen liebevollen Begrüssungen bezeichnet, welche in solchem Falle bei anderen Vögeln in so auffallender Weise Statt finden. Dieser Mangel an gegenseitiger Anhänglichkeit stimmt jedoch mit der allgemeinen Lebenseinrichtung des Kuhvogels überein; denn eine solche Anhänglichkeit würde überflüssig bei ihm sein: da er niemals ein Nest baut oder seine Jungen füttert. Ueberwacht man eine gewisse Anzahl dieser Vögel während der Vermehrungszeit, so bemerkt man: wie das Weibchen seine Gefährten verlässt, ein kränkelndes Ansehen bekömmt und sich auf eine Höhe setzt, von wo es das Treiben der Vögel bei dem Bauen der Nester beobachten kann. Kann es von dem Platze aus keine passende Entdeckung machen, so wird es unruhig und flattert von Baum zu Baum."

Hierauf folgt eine genaue Erzählung darüber, wie einst $P_{0}$ tter ein Weibchen ungefähr eine halbe (englische) Meile weit längs dem Ufer eines Baches in gehöriger, es nicht störender Entfernung beim Nestersuchen begleitete. Da bemerkte er nun: dass es nach allen geeignet scheinenden Dickichten hineinsah, nach dem Legen aber sofort, über den Niederwald hinweg, zu seiner früheren Gesellschaft auf das Feld zurückkehrte.

Auch Nuttall, ein fleissiger und sehr erfahrener praktischer Beobachter, so wie sein Freund Pickering, dem er viel wichtige Mittheilungen verdankt, stimmen hiermit ganz überein. Nachdem N. in seiner Schilderung der Sitten des Kuhfinken daran erinnert hat, dass bei allen übrigen Vögeln, welche den grössten Theil des Jahres hindurch in Gesellschaft leben, die, zum Theil überaus zahlreichen Schaaren der-

*) James Rennie, in der 2ten Ausgabe seiner Schrift über "die Baukunst der Vögcl," deren Original mir nicht zur Hand ist, fasst nach Dr. Frz. Kottenkamp's Uebersetzung (Stuttgart 1847, S. 281-82) die Angabe Pot ter's in der hier folgenden Weise zusammen.

**) Oder, wohl richtiger: was Jenner, (der berühmte Erfinder der Schutzpocken-Impfung,) eigentlich mehr durch Schlüsse gefunden zu haben glaubte, als wirklich "beobachtet" hatte. 
selben zu einer bestimmten Zeit (im Frühlinge) sich auflösen, um sich nach einzelnen Paaren zu vertheilen, fährt er hinsichtlich des Kuhfinken hierüber fort:

-Bei unserem Vogel dagegen tritt eine solche Zeit niemals ein; die Gesellschaften leben und bleiben bei einander, ohne sich jemals zu verpaaren. Es herrscht bei ihnen ein ganz allgemeines Begattungsverhältniss, (A general concubinage prevails among them,) welches kaum irgend welche Eifersucht hervorruft und von keiner dauernden Anhänglichkeit begleitet ist". *)

Man kann diess natürlich immerhin als „P oly andrie der Weibchen, “ im Gegensatze zu der „Polygamie der Männchen“ bei manchen Hühnern etc., bezeichnen.

Bei den K u ckuken stellt sich das aber ganz und gar anders.

Wenn also die amerikanischen Ornithologen hierbei auf den europäischen Kuckuk verweisen, und wenn ebenso R e n n i e bei diesem wieder an den amerikanischen Kuhvogel erinnert: so haben sie natürlich wohl in der Einen Hauptsache beiderseits Recht; nämlich insofern weder der Kuhfink, noch der Kuckuk, selbst nistet, brütet, Junge füttert, u. s. w. Aber sie irren gewiss beiderseits, wenn sie, verleitet durch den berühmten Dr. Je nner, mehr oder weniger auch geneigt sind, anzunehmen : die Weibchen unseres Kuckuks, ebenso wie die , anderer KuckuksArten des alten Festlandes, " lebten gleichfalls, ähnlich denen des Kuhfinken, in beliebiger und ganz ungeordneter Polyandrie. Jenner, der nicht bloss ein denkender Arzt, sondern auch theilweise ein tüchtiger, fachlicher Naturforscher war, hat das allerdings gemeint; nur hat er gewiss es nicht eben durch genaue, anhaltende praktische Beobachtungen herausgebracht, sondern offenbar nur durch Schlüsse zu finden geglaubt, als die Fortpflanzungsgeschichte unseres Kuckuks ihn beschäftigte. $\left.{ }^{* *}\right)$ Indess hat er die Sache wohl auch schwerlich so als bestimmte, erfahrungsmässige Thatsache hingestellt, wie die Wenigen, welche sich für diesen Punkt auf ihn berufen, diess anzusehen scheinen. Und Letztere, wenn sie geneigt sind, bei den Kuckuksweibchen eine der des Kuhfinken ähnliche „Polyandrie“ vorauszusetzen, übersehen dann hierbei einen weiteren Hauptpunkt, welcher bei dem Kuckuke offenbar dagegen spricht: während allerdings beim Kuhfinken die entgegengesetzte Eigenschaft dafür spricht.

Diess ist nämlich eben die ganz eigenthümliche Geselligkeit des Kuhfinken, die eine so unabänderlich dauernde zu allen Zeiten des Jahres bleibt, wie man sie bisher noch bei keinem anderen Vogel kennt. Ganz ihr Gegensatz ist die, im hö chsten Grade Statt findende Ungeselligkeit der Kuckuke, die bei ihnen lediglich für die Dauer der Begattungszeit und zu dem Zwecke der Begattung so weit aufhört, wie diess alsdann unbedingt erforderlich wird: nämlich in so weit, dass sie zwar eben dann, aber (gleich den Spechten) auch zu

*) Manual Ornith. Unit. Stat. I, p. 180.

**) Er war namentlich von Allen der Erste, der (bereits i. J. 1787) bestimmte Versuche, ähnlich wie späterhin bei uns Hr. Pastor B r hm, darüber anstellte: dass, und wie, der junge Kuckuk seine Stiefgeschwister aus dem Neste wirft. 
keiner anderen Zeit, paarweise Gemeinschaft pflegen. Denn späterhin bekümmert sich bekanntlich niemals ein Kuckuk um den anderen, folglich auch kein Gatte um jenen anderen, mit welchem er sich fortgepflanzt hat.

Gerade in Bezug auf die erste Grundbedingung aller Fortpflanzung, auf die leichte, stets gesicherte Möglichkeit des Begattens, kehrt sich also für diese beiden Fälle das Verhältniss in den äussersten Gegensatz des einen zu dem anderen um.

Beim Kuhvogel hat jedes Weibchen in jedem Augenblicke, wo es den Trieb dazu fühlt, auch Männchen zur Befruchtung um sich. Es braucht sich, recht buchstäblich, gar nicht erst nach einem solchen umzusehen: eben so wenig, wie diese es nöthig haben, jemals nach Weibchen zu suchen. Wie aber würde es mit der "Sicherung dieser Möglichkeit" bei dem Kuckuke, diesem entschiedensten Muster von Ungeselligkeit, stehen, w e n n bei ihm gleichfalls eine Polyandrie der Weibchen, d, h. ein völlig ungeregeltes Verhältniss der Geschlechter zu einander selbst in der Fortpflanzungszeit, wirklich bestände? Wer könnte wohl überhaupt glauben, dass es die Natur hierin je so rein auf blossen Zufall könnte ankommen lassen? Oder wer möchte vollends gar annehmen, dass sie das gerade bei dem Kuckuke gethan haben sollte? bei ihm, für dessen gesicherte Vermehrung sie in so auffallender Weise Vorkehrungen der eigenthümlichsten Art getroffen hat: Einrichtungen, die, wie der beispiellose Wechsel in der Färbung seiner Eier und sogar in deren Zeichnung, mit Recht immer mehr unsere Bewunderung erregen, je genauer wir sie kennen lernen.

Einen zweiten, kaum weniger ins Gewicht fallenden Gegensatz und Gegengrund bildet aber noch der, von den Ornithologen Amerika's ausdrücklich hervorgehobene und nach den obwaltenden Verhältnissen auch leicht erklärliche Mangel von Eifersucht beim Kuhfinken.

Ihm steht wiederum die, auf's Höchste getriebene Eifersucht beim Kuckuke gegenüber: da er hierin für die Dauer der Begattungszeit nicht bloss hinter keinem anderen Vogel zurücksteht, sondern eher wohl alle die übrigen bei uns noch übertrifft. Auf Seiten der Männchen ist dieselbe erwiesen durch jene Händel und heftigen Kämpfe, in welche sie gerathen, sobald eines vou ihnen das, allerdings nicht selten vorkommende Ueberschreiten der Gränzen seines Revieres durch ein benachbartes gewahrt.

Aber selbst von Seiten der We ibchen ist sie, obgleich in Folge ihres mehr stillen Verhaltens nicht in so bestimmter Weise beobachtet, doch als wahrscheinlich zu betrachten. Jedenfalls bleibt sie ungleich naturgemässer vorauszusetzen, als das Gegentheil. Denn, wenn sie bei anderen Vögeln hauptsächlich den Männchen eigen ist, so mangelt sie doch auch bei ihnen den Weibchen keineswegs etwa ganz. Ins Besondere tritt sie bei diesen in der Nähe ihrer Nester hervor: indem hier keines die Gegenwart anderer Weibchen, die zufällig einmal dahin kommen, dulden mag. Nun ist zwar nicht gerade anzunehmen, dass ein Kuckuksweibchen die von ihm gesuchten Nester anderer Vögel, deren es sich zum Unterbringen seiner Eier bedienen kann, will und muss, 
ohne Weiteres in gleichem Grade als sein Eigenthum betrachten möchte, wie deren Erbauer selbst es thun; in ziemlich ähnlichem Grade aber thut es diess wohl höchst wahrscheinlich immer schon von vornherein, also vom Beginne seiner Fortpflanzungszeit an, für den von ihm bewohnten Bezirk. Dass jedoch eben dieses Gefühl einer gewissen Art von Besitz oder Mitbesitz und Besitzrecht ihm jedenfalls bei denjenigen Nestern, in die es wirklich gelegt hat, äusserst nahe liegt, ist ja längst mehr als hinreichend erwiesen. Dafür spricht sein wiederholtes Besuchen und genaues Ueberwachen derselben. Vor Allem jedoch zeugt davon sein instinctives, gewaltsames Schalten mit den ausgebrüteten Jungen der wirklichen Eigenthümer : da es diese gewöhnlich selbst in freistehenden, offen-gebauten Nestern schon herausnimmt und fortträgt, bevor sie noch der junge Kuckuk selbst herauszudrängen vermag; ebenso, wie es sie aus geschlossenen Nestern und Nisthöhlen, wo letzterer sie gar nicht herauszuwerfen im Stande sein würde, allein fortschafft.

Mit dem Allem würde es gewiss durchaus nicht zusammenstimmen, wenn man annehmen wollte: das Kuckuksweibchen sollte nicht in wenigstens "ähnlicher", wenn auch vielleicht nicht gleicher Weise Eifersucht gegen andere hinsichtlich der Vogelnester hegen, wie die Männchen sie gegen einander in Betreff des Besitzes von Weibchen fühlen.

Vielmehr würde es diess gewiss sogar in dem Falle nicht thun oder thun können, wenn es wirklich in Polyandrie lebte, sich also nach Belieben heut an dieses, morgen an jenes Männchen hielte, wie und wo $\mathrm{ihm}$ deren eines begegnete. Es würde nämlich für diese Frage noch immer Nichts gewonnen sein, wenn man wirklich die, in jeder Beziehung unhaltbare Theorie annehmen wollte: dass ,jedes Kuckuksweibchen regelmässig nur in die Nester irgend Einer bestimmten Sängerspecies legen" solle, (weil vermeintlich ,jedes immer gleichcharacterisirte Eier legen" soll!) Damit würde nur eine neue und gewiss höchst seltsame, schwer zu lösende Schwierigkeit eintreten. Denn wie sollte dann ein Kuckuks-Weibchen es dem anderen wohl ansehen, ob dieses Nester derselben Vogelart brauche und suche? oder ob es denen, einer bestimmten" anderen Art nachgehe, die jenes nicht brauchen könnte, weil seine Eier zu denen dieser "anderen Art" nicht passten? In dem letzteren Falle würde freilich eines dem Bedürfnisse des anderen keinen Schaden thun. In dem ersteren dagegen würde es höchst wahrscheinlich tüchtige Händel zwischen ihnen geben. Denn es dürfte von den zwei, oder sehr häufig mehreren „Concurrentinnen" wohl schwer vorauszusetzen bleiben: dass sie Neigung oder Mittel haben sollten, sich in friedlicher Weise über diese, für sie allerseits "erste" Grundfrage mit einander zu verständigen. ")

Bei den, in solchen "Umgangs-Beziehungen" vollständig ,emancipirten" Kuhfinkinnen dagegen, diesen thatsächlichen Urbekennerinnen der sauberen Lehren St. Simon's, fällt auch jede solche Schwierigkeit aus doppeltem Grunde hinweg.

*) Jene ganze Theorie erschöpft aberhaupt weder die Regel, noch erKlärt sie irgend eine A us nahme. Die Ansicht des Hrn. Kunz dagegen ( „Naumanma, Jahrg. 1850, Heft II, S. 51 ff.) thut auf gleich einfache Weise Beides. Und nur so Elwas nennt man "naturgemäss." 
Erstens legen sie nämlich, so viel bisher schon bekannt, ganz nach Belieben in die Nester von einem vollen Dutzende verschiedener, kleinerer Vogelarten. Schon desshalb also kommen sie einander, bei so grosser Auswahl, nicht leicht in den Weg. Zweitens legen sie alle mit einander so ähnliche Eier, wie andere Vögel einer und derselben Art; nicht aber so überaus verschiedene, wie unsere Kuckuksweibchen. Die ihrigen bleiben daher "überall, sowohl durch ihre nicht-passende Färbung und Zeichnung, wie durch ihre weit überlegene Grösse vor jenen der Nesteigenthümer, sofort kenntlich". *) Folglich werden sie auch gewiss ebenso für die Kuhfinken erkennbar sein; und mithin wird kein Weibchen von ihnen füglich auch nur in die Gefahr kommen können, in ein Nest zu legen, in welches bereits ein anderes diess vor ihm gethan hat.

Hieraus ersieht man leicht, wie unrichtig es war oder sein würde, vom Kuhfinken wegen der gemeinschaftlichen Hauptsache, des Nichtbrütens, ohne Weiteres auch hinsichtlich der Nebensachen auf die Kukkuke schliessen zu wollen, oder umgekehrt.

Was dagegen um so untrüglicher bleiben wird, das ist der Schluss: dass bei den Kuckuken ein fortwährender Streit und Krieg, wie derselbe im Falle einer Polygamie oder Polyandrie, und noch mehr natürlich vollends erst durch beide zusammen, vermöge der Eifersucht würde entstehen müssen, offenbar nur von höchst nachtheiligen Folgen auf das gesammte Fortpflanzungsgeschäft würde sein können. Eben desshalb wird aber gewiss die Natur hier keines von Beidem eingeführt haben. In der That hat bisher auch, so viel mir bekannt, von unseren tüchtigsten deutschen ornithologischen Praktikern keiner Etwas von Beidem wahr- oder angenommen. * ${ }^{*}$ ) Dagegen würde nach der Theorie des Hrn. Baldamus offenbar nur die Annahme einer gleich allgemeinen Polyandrie, wie sie beim Kuhfinken Statt findet, das einzige Mittel bleiben, um di e Erscheinung zu erklären: dass überall Kuckuks-Eier der verschiedensten Färbungen dicht bei, wenn auch gewöhnlich erst mehr oder weniger $\mathrm{nach}$ einander gefunden werden. Denn wenn eben "jedes Weibchen immer gleichcharacterisirte Eier" legte: dann liesse sich jene Verschiedenheit nur dadurch erklären, dass jedes Weibchen auf den Revieren beliebig vieler Männchen umherschweifte, die sich dann freilich auch gern mit ihm begatten würden. -

Uebrigens sind aber, nebenher gesagt, Polygamie und Polyandrie auch nur verschiedene Abstufungen eines und desselben unbestimmten geschlechtlichen V erhältnisses. Ihrem Wesen nach fallen sie daher im Grunde wiederum zusammen. Denn überall, wo es z. B. viel Auerhühner, Birkhühner oder Fasane giebt, und wo man die

*) Es trift sich in der That ungemein hübsch, dass Wils on diess ausdrücklich mit anführt. Er hat also damit lange zum Voraus eine Frage beantwortet, an die man erst jetzt gedacht haben würde, um sie aufzustellen.

**) Ich wenigstens erinnere mich nur Eines Berichtes über einen solchen, wirklich beobachteten Fall. Dergleichen kommen jedoch auch sonst zuweilen vor; und beim Kuckuke, wo die Männchen ofter weggeschossen werden, als die sich weniger selbst verrathenden Weibchen, kann der Zufall wohl so Etwas desto leichter herbeiführen. 
Hähne nicht allzu sehr wegschiesst, giebt es gewiss nicht Eine Henne, die nicht jeden Frühling hindurch mehr oder weniger in Polyandrie lebte oder geriethe. Jede sucht und findet ja nur gewöhnlich, aber nicht immer, denselben Hahn wieder auf; besonders nicht gegen das Ende der Balzzeit. Denn alsdann treten ohnehin die ,abgebalzten " (matter gewordenen) alten mehr oder weniger von selbst zurück; und jüngere treten dann an deren Stelle ein.

In Bezug auf das Paaren, Brüten und Pflegen oder Führen der Jungen aber wird es dem zufolge heissen:

Bei den monog amen Vögeln "pflegen" die Männchen stets mit; und bei den meisten brüten sie auch mehr oder weniger.

Bei den wenigen polygamen, wo meistens das Pflegen ohnehin wegfällt, bekümmern sich die Männchen auch nicht um das Brüten und Führen der Jungen: weil sie sich überhaupt nicht paaren.

Bei den monogamen Kuckuken brüten und pflegen weder die Weibchen, noch die Männchen: obgleich sie wohl ohne Zweifel sich paaren.

Nur beim $\mathrm{Kuhfinken}$ allein findet Nichts von dem Allem zusammen Statt.

Berlin, den 10. September 1853.

\section{Der grosse Würger (Lanius excubitor Lin.) und einige seiner Verwandten.}

\section{Von \\ Pastor Ch. Ludwig Brehm.}

Ich habe in meinem Handbuche S. 232 von dem Lanius exubitor 2 verschiedene Bildungen als 2 Subspecies aufgeführt, allein ich habe mich überzeugt, dass es richtige Species sind, was auch durch gepaarte Paare, da die zu einem jeden gehörenden Vögel sich völlig gleichbleiben, bewiesen wird. Ferner habe ich bei den Würgern zwei $\mathbf{A b}-$ theilungen gemacht und die eine

Raubwürger, Lanii rapaces

genannt. Auch diese Annahme hat sich bewährt, denn die hierher gehörigen haben nicht nur eine andere Schnabelbildung, (derjenigen entsprechend, welche die auf den Raub von Säugethieren und Vögeln angewiesenen Tagraubvögel, besonders die Habichte zeigen, während die Schnabelbildung der insectenfressenden Würger jener der von Insecten lebenden Falken ähnelt,) sondern auch eine durchaus räuberische Natur, welche so weit geht, dass einst bei mir ein Bruder seine Schwester in der Gefangenschaft nach langem Kriege tödtete und auffrass. Deswegen heissen auch diese Vögel nicht nur $W u ̈ r g e r$, sondern auch in manchen Gegenden Deutschlands Neuntödter, weil die Volkssage behauptet, sie brächten in einem Tage 9 Vögel um. Hierher gehören : 


\section{$2 \mathrm{BHL}$ Biodiversity Heritage Library}

1854. "Das geschlechtliche Verhältniss bei den nicht selbst brütenden Vögeln." Journal

$f u$

r Ornithologie 2, 137-143. https://doi.org/10.1007/bf02006767.

View This Item Online: $\underline{\text { https://www.biodiversitylibrary.org/item/49195 }}$

DOI: https://doi.org/10.1007/bf02006767

Permalink: https://www.biodiversitylibrary.org/partpdf/142197

\section{Holding Institution}

Natural History Museum Library, London

\section{Sponsored by}

Natural History Museum Library, London

\section{Copyright \& Reuse}

Copyright Status: Public domain. The BHL considers that this work is no longer under copyright protection.

This document was created from content at the Biodiversity Heritage Library, the world's largest open access digital library for biodiversity literature and archives. Visit BHL at https://www.biodiversitylibrary.org. 